\title{
Intestinal Helminthic Infection Increases Serum Levels of IL-2 and Decreases Serum TGF-Beta Levels in Nigerian Asthmatic Patients
}

\author{
Ganiyu 0. Arinola1, Oluwafemi Oluwole², Regina Oladokun'1, Babatunde 0. Adedokun', \\ Olufunmilayo I. Olopade ${ }^{2}$, Christopher 0. Olopade ${ }^{3 *}$ \\ ${ }^{1}$ College of Medicine, University of Ibadan, Ibadan, Nigeria \\ ${ }^{2}$ Center for Clinical Cancer Genetics and the Center for Global Health, University of Chicago, Chicago, USA \\ ${ }^{3}$ Department of Medicine and the Center for Global Health, University of Chicago, Chicago, USA \\ Email: solopade@bsd.uchicago.edu
}

Received 10 December 2013; revised 10 January 2014; accepted 18 January 2014

Copyright (C) 2014 by authors and Scientific Research Publishing Inc.

This work is licensed under the Creative Commons Attribution International License (CC BY).

http://creativecommons.org/licenses/by/4.0/

c) (i) Open Access

\section{Abstract}

Background: Asthma is less common in countries outside the organization for economic cooperation and development (OECD). One suggested mechanism for this disparity is the early life exposure to helminthic infection in non-OECD countries due to predominant Th1 immune response, which down-regulates Th2 responses required for exacerbation of asthma. This report is the first in the literature to describe the effects of helminthic infection on immune responses in asthmatic children compared to non-asthmatic controls. Methods: We administered the International Study of Asthma and Allergies in Childhood (ISAAC) questionnaire to 1690 high school students in three rural communities in southwestern Nigeria. Based on questionnaire responses and medical examination, identified asthma cases were matched with controls. Stool samples were collected from all subjects and screened for intestinal helminthic infection. The serum of 12 asthmatics and 12 non-asthmatics (6 with intestinal helminthic infection and 6 without intestinal helminthic infection in each group) were assayed for interleukin (IL)-2, IL-4, IL-5, IL-6, IL-10, IL-12, IL-13, IL-17A, interferon-gamma (IFN- $\gamma$ ), tumor necrosis factor-alpha (TNF- $\alpha$ ), granulocyte macrophage colony-stimulating factor (GM-CSF), and transforming growth factor-beta (TGF- $\beta$ ). Results: The mean $( \pm S D)$ serum levels of IL-2 significantly increased in asthma cases with intestinal helminthic infection compared with asthma cases without infection $(41.5 \pm 9.7 \mathrm{vs.} 29.7 \pm 10.3 ; p=0.035)$, controls with intestinal helminthic infection compared with cases without infection $(52 \pm 4.7 \mathrm{vs} .29 .7 \pm 10.3$; $p<0.001$ ), and controls with intestinal helminthic infection compared to controls without infec-

*Corresponding author.

How to cite this paper: Arinola, G.O., Oluwole, O., Oladokun, R., Adedokun, B.O., Olopad, O.I. and Olopade, C.O. (2014) Intestinal Helminthic Infection Increases Serum Levels of IL-2 and Decreases Serum TGF-Beta Levels in Nigerian Asthmatic Patients. Open Journal of Immunology, 4, 1-8. http://dx.doi.org/10.4236/oji.2014.41001 
tion (52 \pm 4.7 vs. $34.2 \pm 10.4 ; p=0.003$ ). Mean levels of TGF- $\beta$ significantly increased in controls without infection compared with asthma cases with infection $(1833 \pm 93.1$ vs. $633.3 \pm 294.4 ; p<$ 0.001), controls without intestinal helminthic infection compared with asthma cases without infection $(1833 \pm 93.1$ vs. $916.7 \pm 204.1 ; p<0.001)$, controls with intestinal helminthic infection compared to controls without infection $(2366.7 \pm 760.7$ vs. $1833 \pm 93.1 ; p<0.001)$, and controls with intestinal helminthic infection compared with asthma cases without infection $(2366.7 \pm$ 760.7 vs.916.71 \pm 204.1; $p=0.007$ ). Conclusion: Intestinal helminthic infection independently increases IL-2 levels, while asthma decreases the level of TGF- $\beta$, which is further depressed by intestinal helminthic infection.

\section{Keywords}

Intestinal; Helminthic; Cytokines; Immune Responses; Asthma; Children; Nigeria

\section{Introduction}

Early studies on disease distribution suggested that asthma is less common in countries outside the organization for economic cooperation and development (OECD), especially in rural communities [1] [2]. The reason for the regional difference is not clearly understood, but infection with intestinal helminthes has been postulated as an important factor in determining prevalence through various mechanisms that may suppress or inhibit the immune responses which lead to clinical symptoms of asthma [3]. While eradication of parasites may offer many public health benefits, the hypothesis that helminthic infections protect against allergic disease raises the possibility that eradication may, in fact, increases the incidence of asthma and allergic diseases. Therefore, it is important to establish whether helminthic infection and asthma are related.

The immune response mounted to helminthic infections is characterized by T-helper type 2 (Th2), which is thought to be protective [4]. There is also evidence, however, that these parasites might enhance their own survival by modulating the immune responses of their host and inducing regulatory responses that dampen activity of effector cells [5]. Such responses typically involve increased production of cytokines, such as interleukin IL-4, IL-5, IL-9, IL-10 and IL-13, allergen-specific and total immunoglobulin E (IgE), eosinophilia, tumor necrosis factor-alpha (TNF- $\alpha$ ), transforming growth factor-beta (TGF- $\beta$ ) and epidermal growth factor (EGF) [6] [7]. Cytokines are usually extracellular signaling proteins produced by many cell types that are involved in cell-to-cell interactions acting through specific receptors on the surface of target cells [8]. They play an integral role in the coordination and persistence of the inflammatory process in the airways of asthmatics, as they are capable of inducing many of the pro-inflammatory effects characteristic of the disease [9]. Th1 cytokines (e.g. IFN- $\gamma$, TGF- $\beta$, etc.) enhance cellular immune responses while Th2 cytokines (IL-4, IL-10, IL-13, etc.) favor antibody responses. These two sub-sets inhibit one another [10], so cytokine dysregulation has been under intensive study for its possible role in the pathogenesis of many diseases [10].

Allergic diseases including asthma, eczema, and rhinitis are associated with Th-2 inflammation [11]. In individuals predisposed to allergic disease, inflammation mediated by helminthic infection can be modulated by the host immune response and that failure of the expression of similar mechanism may be responsible for the clinical expression of allergic disease [6] [12]. Although insight into the pathophysiology of asthma has increased substantially in industrializedcountries, the relationship between helminthic infections and asthma risk, particularly in rural areas of non-OECD countries remain to be further clarified. The "hygiene hypothesis" suggests that improved sanitation leads to an increased incidence of allergic disease in susceptible individuals [13]. The situation is different in non-OECD countries, such as sub-Saharan Africa, Asia, and Latin America, where helminthic infections remain endemic and the risk of developing allergic diseases, like asthma, is lower [14] [15]. Several studies have shown a negative correlation between chronic helminthic infections and allergic reactivity to common allergens [10] [13] [16]. There is also growing evidence that intestinal helminthic infection may be capable of modulating the expression of allergic disease [16].

Apart from the fact that Th2 response has evolved to minimize the virulence of helminthes [4], Th2 responses also protect against allergies and asthma, limit the number of helminths in the intestinal tract [13] and repair 
tis-sue damage caused by the helminths that have colonized the intestine [17]. In addition to the Th2 responses during helminthic infection regulatory B cells, alternatively activated macrophages (or M2) were key components of the immune regulatory network functioning during helminthic infections [18].

Based on previous studies, it is clear that the interplay between helminthic infections and allergies is complex and the results are inconsistent. Identification of a link between helminthic exposure and down-regulation of Th2 immune response may suggest promising avenues for future investigation into the etiology or the mechanisms responsible for triggering of asthma. Therefore, the serum levels of 12 cytokines (IL-2, IL-4, IL-5, IL-6, IL-10, IL-12, IL-13, IL-17A, IFN- $\gamma$, TNF- $\alpha$, GM-CSF, and TGF- $\beta$ ) were examined in asthma patients with or without intestinal helminthic infection compared with non-asthmatics with or without helminthic infection. The aim of the study was to establish whether or not on-going intestinal helminthic infection affects serum cytokine levels in a cohort of asthmatic and non-asthmatic Nigerian school children in rural areas. To our knowledge, no previous study has investigated a wide panel of cytokines in asthmatics with and without intestinal helminthic infection. Additionally, no such studies have been conducted in Nigerian asthmatics.

\section{Methods}

The Institutional Review Board on Human Research at the University of Ibadan, Nigeria, and the University of Chicago, USA, approved the research protocol. Approval to conduct the study was also obtained from the Oyo State Ministry of Education, Ibadan, Nigeria. The parents or guardians of all participants provided written informed consent and the students provided assent for participation in the study.

\subsection{Study Participants}

The study was conducted in Abanla, Eruwa and Igbo-Ora, three rural communities in southwestern Nigeria. The prevalence of asthma in this region in the past 2 years in school children aged 13 - 14 years reported in a previous study was 7.2\% [19]. The study population included apparently healthy 24 children (12 asthmatics and 12 non-asthmatics) of similar ages and gender (Table 1), which is a subset of the 1690 children screened with ISAAC questionnaires designed to diagnose patients with asthma.

\subsection{Asthma Screening}

Children were screened for asthma based on the affirmative responses to the ISAAC questionnaire which includes questions on symptoms of asthma and/or physician's diagnosis of asthma. Asthma was defined by a positive response to the question "Has a doctor ever told you that you have asthma?" Those that reported non-physician diagnosed asthma were further assessed based on their "Asthma-related symptom" if they reported a positive response to either "Have you ever had wheezing or whistling in the chest at any time in the past 12 months?" or "In the past 12 months have you had a dry cough at night, apart from a cough associated with a cold or chest infection?” Those that reported "No" responses to both questions on physician-diagnosed asthma and asthmarelated symptoms were classified as non-asthmatic controls. Based on questionnaire responses and detailed medical examination by a physician, 104 asthmatics were identified and were subsequently matched with 121 non-asthmatic controls for a total of 225 subjects. Stool samples were collected from all asthmatics and controls. Of the 225 subjects, 12 asthmatics and 12 non-asthmatics (6 with intestinal helminthic infection and 6 without intestinal helminthic infection in each group) were assayed for IL-2, IL-4, IL-5, IL-6, IL-10, IL-12, IL-13, IL-17A, IFN- $\gamma$, TNF- $\alpha$, GM-CSF, and TGF- $\beta$. For analysis, data obtained on the 24 (12 asthmatics and 12 non-asthmatic controls) subjects matched for age, gender and intestinal helminthic infection were used. Excluded from the study are those on medication, obviously sick and those with malaria parasites in blood film.

Table 1. Age and gender distribution of the subjects.

\begin{tabular}{ccccc}
\hline & Asthma (+H) & Asthma $(-\mathbf{H})$ & Control $(-\mathbf{H})$ & Control $(+\mathbf{H})$ \\
\hline Age $($ Mean \pm SD) & $14.83 \pm 1.83$ & $14.00 \pm 2.89$ & $13.83 \pm 2.56$ & $13.00 \pm 2.97$ \\
Gender (M/F) & $5 / 1$ & $4 / 2$ & $5 / 1$ & $5 / 1$ \\
\hline
\end{tabular}

$+\mathrm{H}=$ with intestinal helminthic infection; $-\mathrm{H}=$ without intestinal helminthic infection. 


\subsection{Stool Evaluation for Parasites}

All subjects produced fresh stool samples that were examined for ova and parasites at the parasitology laboratory at the University College Hospital, Ibadan, Nigeria using the previously described procedure at the parasitology laboratory at the University College Hospital, Ibadan, Nigeria [19]. These ova were used to identify the types of adult helminthic parasite present in the intestine. Labeled dried, leak proof, clean plastic containers were distributed for the collection of fresh fecal samples which were microscopically examined for characteristic ova of intestinal parasites.

\subsection{Blood Sample and Cytokine Analysis}

Venous blood samples were collected from all subjects in 10ml Vacutainer EDTA tubes. All samples were processed at the Institute for Advanced Medical Research and Training (IMRAT) laboratory at the College of Medicine, University of Ibadan, Nigeria. Serum samples were frozen and stored at $-80^{\circ} \mathrm{C}$ until analysis was performed in the Immunology Unit, Department of Chemical Pathology, University of Ibadan, Nigeria. Samples were analyzed for the levels of 12 serum cytokines (IL-2, IL-4, IL-5, IL-6, IL-10, IL-12, IL-13, IL-17A, IFN- $\gamma$, TNF- $\alpha$, GM-CSF, and TGF- $\beta$ ) using Multi-Analyte ELISArray Kits based on the method described by the manufacturer (SA Biosciences, America) as follows: $50 \mu \mathrm{l}$ per well of appropriate sample dilution buffer, antigen standard cocktail or an experimental sample was pipetted into microtiter plates. This sample was incubated at room temperature $\left(25^{\circ} \mathrm{C}-27^{\circ} \mathrm{C}\right)$ for 120 minutes. The ELISA immunoplate was washed 3 times with $350 \mu 1 /$ well of washing buffer. After washing, $100 \mu \mathrm{l}$ per well of detection antibodies was added. This mixture was incubated at room temperature for 60 minutes. The immunoplate was rewashed 3 times with $350 \mu 1 /$ well of washing buffer. A concentration of $100 \mu \mathrm{l} /$ well of diluted Avidin-HRP conjugate was added, after which the plate was incubated at room temperature for 30 minutes in darkness. The plate was washed 4 times and $100 \mu \mathrm{l}$ per well of developing solution was added. The reaction was stopped with $100 \mu 1 /$ well of Stop Solution and the optical density (OD) was read at $450 \mathrm{~nm}$ within 30 minutes following the addition of stop solution. The average absorbance value of each OD was plotted against corresponding cytokine values to create a standard curve. The average absorbance of each serum sample was used to determine corresponding cytokine values by interpolating from the curve.

\subsection{Data Analysis}

Data were analyzed using SPSS and the values were presented as mean $( \pm$ SD). Mean $( \pm$ SD) between and within groups were compared using ANOVA and Student $t$-test. $p$-value $\leq 0.05$ was reported as statistically significant.

\section{Results}

The result shows that there were no significant differences between serum levels of IL-4, IL-5, IL-6, IL-10, IL-12, IL-13, IL-17A, IFN- $\gamma$, TNF- $\alpha$ and GMCSF when all groups were compared (Table 2). Only IL-2 and TGF- $\beta$ showed significant differences. Considering IL-2, significant increases were found between asthma cases with intestinal helminthic infection compared with asthma cases without infection $(p=0.035)$, controls with intestinal helminthic infection compared with cases without infection $(p<0.001)$, and controls with intestinal helminthic infection compared with controls without infection $(p=0.003)$. This result shows that intestinal helminthic infection influenced the increase in IL-2 levels more than asthma. In terms of TGF- $\beta$, significant increases were found between controls without intestinal helminthic infection compared with asthma cases with infection $(p<0.001)$, controls without intestinal helminthic infection compared with asthma cases without infection $(p<0.001)$, controls with intestinal helminthic infection compared to controls without infection $(p<$ $0.001)$, and controls with parasites compared with asthma cases without infection $(p=0.007)$. This result shows that asthma decreases the level of TGF- $\beta$ and this was further depressed by intestinal helminthic infection.

Both IL-2 and TGF- $\beta$ were increased in non-asthmatic controls with intestinal helminthic infection compared with asthma cases without infection or non-asthmatic controls with intestinal helminthic infection compared with non-asthmatic controls without infection. 
Table 2. Comparison of mean cytokine levels between asthma cases and non-asthmatic controls.

\begin{tabular}{cccccc}
\hline & $\begin{array}{c}\text { Asthmatics (+H) } \\
(\mathbf{N}=\mathbf{6}) \text { Mean } \pm \text { SD }\end{array}$ & $\begin{array}{c}\text { Asthmatics (-H) } \\
(\mathbf{N}=\mathbf{6}) \text { Mean } \pm \text { SD }\end{array}$ & $\begin{array}{c}\text { Controls (+H) } \\
(\mathbf{n}=\mathbf{6}) \text { Mean } \pm \text { SD }\end{array}$ & $\begin{array}{c}\text { Controls (-H) } \\
(\mathbf{n}=\mathbf{6}) \text { Mean } \pm \text { SD }\end{array}$ & $p$-value \\
\hline IL-2 & $41.5 \pm 9.7$ & $29.7 \pm 10.3$ & $52 \pm 4.7$ & $34.2 \pm 10.4$ & $0.002^{*}$ \\
IL-4 & $21.7 \pm 9.3$ & $20.8 \pm 10.2$ & $22.5 \pm 8.8$ & $14.2 \pm 2.0$ & 0.309 \\
IL-5 & $21.7 \pm 9.3$ & $19.7 \pm 10.0$ & $16.7 \pm 2.6$ & $14.7 \pm 3.3$ & 0.359 \\
IL-6 & $15.5 \pm 4.9$ & $14.0 \pm 4.6$ & $12.2 \pm 3.9$ & $12.2 \pm 3.9$ & 0.501 \\
IL-10 & $17.5 \pm 7.5$ & $12.2 \pm 3.9$ & $13.5 \pm 5.1$ & $12.2 \pm 3.9$ & 0.281 \\
IL-12 & $15.7 \pm 8.0$ & $12.7 \pm 4.1$ & $10.0 \pm 0.6$ & $10.8 \pm 4.6$ & 0.254 \\
IL-13 & $25.0 \pm 7.7$ & $22.7 \pm 8.6$ & $20.8 \pm 2.0$ & $21.0 \pm 4.7$ & 0.648 \\
IL-17A & $74.2 \pm 12.8$ & $95.8 \pm 52.6$ & $77.5 \pm 17.5$ & $111.7 \pm 68.5$ & 0.450 \\
IFN- $\gamma$ & $15.8 \pm 2.0$ & $15.5 \pm 3.4$ & $15.0 \pm 3.2$ & $14.7 \pm 4.1$ & 0.927 \\
TNF- $\alpha$ & $19.7 \pm 7.1$ & $20.2 \pm 4.1$ & $20.0 \pm 7.1$ & $21.3 \pm 4.3$ & 0.963 \\
GM-CSF & $25.0 \pm 17.3$ & $41.7 \pm 33.6$ & $48.3 \pm 34.7$ & $19.2 \pm 2.0$ & 0.197 \\
TGF- $\beta$ & $633.3 \pm 294.4$ & $916.7 \pm 204.1$ & $2366.7 \pm 760.7$ & $1833 \pm 93.1$ & $<0.001^{* *}$
\end{tabular}

$+\mathrm{H}=$ with intestinal helminthic infection; $-\mathrm{H}=$ without intestinal helminthic infection, ${ }^{*}$ Post hoc: Significant differences found between Asthma cases with parasite vs. Asthma cases without parasite $(p=0.035)$; Cases without parasite vs. controls with parasite $(p<0.001)$; controls with parasite vs controls without parasite $(p=0.003)$. ${ }^{* *}$ Post hoc: Significant differences found between cases with parasite vs. control without parasite $(p<0.001)$; cases without parasite vs. controls without parasite $(p<0.001)$; controls without parasite vs. controls with parasite $(p<0.001)$; controls with parasite vs. cases without parasite $(p=0.007)$.

\section{Discussion}

The cytokine basis of hygiene hypothesis in asthmatic patients is reported to be complex and controversial [20]. Hygiene hypothesis in asthma patients could not be explained simply by the failure to shift from Th2 to Th1 immune responses [21]. Asthma is associated with Th2 (IL-4, -5 and -13) cytokine secretion [22] which is modulated by many factors such as pre-existing or co-infections with bacteria, viruses and parasites, autoimmune disorders [23], cytokine environment and other unidentified factors [20]. Furthermore, evidence suggests that suppression of T-regulatory cells may contribute to the underlying immune mechanisms involved in allergy and asthma [20]. The present study investigated the influence of intestinal helminthic infection on cytokine responses in asthma patients and determined if regulatory-T lymphocytes modulate cytokine responses in asthma patients.

Results show that Th2 cytokines (IL-3, -4, -5, -6, -10 and -13) were elevated (non-significantly) in both groups of asthma patients compared with non-asthmatic controls without intestinal helminthic infection but these increases were higher in asthma patients with intestinal helminthic infection compared with asthma patients without infection. This result suggests that asthma stimulates predominantly Th2 cytokine responses, rather than Th1 responses, as previously reported [22]. This predominant Th2 response in patients with asthma may be explained by the fact that dendritic cells, which are the primary link between the immune system and external allergens, process and present peptides of the allergen via MHC II to TCR of progenitor T-cell. Binding between co-stimulatory molecules (B7.2 of dendritic cell and CD28 molecule of progenitor T-cell) lead to proliferation of Th2 cells and secretion of IL-3, -4, -5, -10, -13 and GM-CSF in asthmatics.

In addition, these results demonstrate that intestinal helminthic infection increases the levels of Th2 cytokines in both asthmatic patients and non-asthmatic controls, suggesting that helminthic infections aggravate Th2 responses in asthmatic and non-asthmatic controls. Previous studies reported that the immune response of the host to helminthic infection is dominated by significant production of Th2 cytokines such as IL-4, IL-5, IL-10, IL-13, IL-25, and IL-31 [24]-[27]. Parasite antigens are exo-antigens, which are processed by antigen presenting cells and peptide presented via MHC II for B-cell activation and antibody production, eosinophil differentiation and recruitment. Therefore, helminthic infection is often reported to be associated with high levels of IgE, IgG1 and 
IgG4, eosinophilia and mast cell responses [28]. It is of great interest to determine levels of IgE and IgG subclasses (IgG1 and IgG4) in asthma patients with or without helminthic infections since cytokines such as IL-4, IL-5 and IL-13 interact with their receptors to stimulate IgE production and increased numbers of eosinophilia and mast cell; all of which are capable of inducing inflammation in the respiratory tract [7].

Th2 cytokines are important in asthma patients with intestinal helminthic infection. They stimulate the production of IgE specific for both asthma allergens and intestinal helminthic antigens. These different sets of IgE compete for binding sites on mast cells. Mast cell degranulation, however, requires two or more antigen specific IgE to cross-link. Cross-linking of non-specific IgE will not cause mast cell degranulation and leads to non-secretion of vasoactive amines resulting to desensitization. Parasite specific IgG subclasses with receptors on mast cells may also cause desensitization of mast cells [29] [30]. This study, therefore, provides additional insight into the role of helminthic infection in modulating Th1 and Th2 responses in patients with asthma.

TGF- $\beta$ and IL-2 were significantly different in asthmatic patients with or without intestinal helminthic infection compared with non-asthmatic controls with or without infection. IL-2 is involved in eosinophilia in vivo, growth and differentiation of resting T cells [9]. IL-2 is involved in control of intracellular pathogens and also contributes to inflammatory responses. IL-2 activates and differentiates resting T-cells into either Th1 or Th2 cell type. This phenomenon may explain the elevated IL-2 levels in asthma or non-asthmatic controls with intestinal helminthic infection compared with non-asthmatic controls without intestinal helminthic infection, since allergens and helminthic antigens stimulate Th2 function.

Clearly, dysregulated immune system involved in allergy and asthma cannot be explained simply by Th1/Th2 dichotomy alone [20]. Another mechanism may involve T-regulator/suppressor cells. Several types of Treg cells have been described (natural Tregs, $\operatorname{Tr} 1$ and Th3). Th3 cells make TGF- $\beta$, which inhibits development of both Th1 and Th2 cells [31]. Reduced levels of TGF- $\beta$ in asthma patients may be an allergen-induced mechanism to prevent down regulation of Th2 cytokines in asthmatics. This phenomenon is supported by reduced level of TNF- $\alpha$ (a Th1 cytokine). Th1 cytokine antagonizes Th2 responses. Therefore, raised levels of Th2 cytokine in these subjects might explain the observed low levels of TNF- $\alpha$. In contrary to asthmatics, helminthic infection is capable of inducing regulatory T-cells (Trigs) [28], which may help control morbidity and dampen resistance to re-infection through their potent immune regulatory mechanisms. Up-regulation of TGF- $\beta$ production has been described in individuals with chronic Schistosoma infection [32]. Similarly, the raised levels of TGF- $\beta$ in nonasthmatic controls with intestinal helminthic infection compared with controls or asthmatics without intestinal helminthic infection are most likely influenced by the chronic helminthic infection. This observation raises the need to relate cytokine levels with different severities of helminthic infection in asthmatics.

A major limitation of this study is the relatively small number of asthmatic and non-asthmatic subjects studied. This small sample size was due to a continuous deworming program among school children by the Federal Government of Nigeria, rigorous sex/age matching of asthmatic and non-asthmatic subjects for this study, and the selection of subjects with only helminthic infection. Thus, there is need for a much larger study to further establish the postulated causal links and to investigate the influence of anthelminthic treatment on immune response, atopy and allergic disorders. Different species of parasite infection also may have important effects on the pathogenesis of asthma. This study did not assess specie-specific parasite infection, instead using pooled intestinal parasite infection data. This choice limits the ability to clearly establish if the relationships between intestinal helminthic infection and asthma cytokines (IL-2 and TGF- $\beta$ ) observed in this study are likely to be parasite specie-specific or parasite-intensity related. The potential modulatory influence of specific parasite species on asthma development and expression warrants further investigation. In addition, determination of intestinal helminthic specific IgE and allergen specific IgE in asthma patients with or without intestinal helminthic infection will also further improve our understanding of the relationship between helminthic infection and asthma modulation.

\section{Conclusion}

Using a wide panel of cytokines in asthmatics with and without intestinal helminthic infection, the present study reported significantly raised levels of IL-2 in asthma patients with intestinal helminthic infection and non-asthmatic controls with intestinal helminthic infection compared with non-asthmatic non-infected controls and reduced levels of TGF- $\beta$ in asthmatics with or without intestinal helminthic infection compared with controls without intestinal helminthic infection. Therefore, IL-2 may be used to differentiate asthma patients with intes- 
tinal helminthic infection from asthma patients without intestinal helminthic infection. Moreover, intestinal helminthic infection potentiates IL-2 production but reduces TGF- $\beta$ level in asthmatics. This result suggests that intestinal helminthes infection promotes pro-inflammatory responses in asthma patients. The role of TGF- $\beta$ in intestinal helminthic-based hygiene hypothesis needs further study.

\section{Acknowledgements}

The authors are grateful to Ralph and Marion FALK Medical Trust, Chicago, USA for funding the project. The authors are also grateful to Healthy Life for All Foundation (HLF), Ibadan, Nigeria, for assistance with project execution and to Rosseau Natalie for editorial assistance.

\section{References}

[1] Asher, M.I., Montefort, S., Bjorksten, B., Lai, C.K., Strachan, D.P., Weiland, S.K., et al. (2006) Worldwide Time Trends in the Prevalence of Symptoms of Asthma, Allergic Rhinoconjunctivitis, and Eczema in Childhood: ISAAC Phases One and Three repeat Multicountry Cross-Sectional Surveys. Lancet, 368, 733-743. http://dx.doi.org/10.1016/S0140-6736(06)69283-0

[2] Weinberg, E.G. (2000) Urbanization and Childhood Asthma: An African Perspective. Journal of Allergy and Clinical Immunology, 105, 224-231. http://dx.doi.org/10.1016/S0091-6749(00)90069-1

[3] Yazdanbakhsh, M. and Matricardi, P.M. (2004) Parasites and the Hygiene Hypothesis: Regulating the Immune System? Clinical Review in Allergy and Immunology, 26, 15-24. http://dx.doi.org/10.1385/CRIAI:26:1:15

[4] Danilowicz-Luebert, E., O’Regan, N.L., Steinfelder, S. and Hartmann, S. (2011) Modulation of Specific and Allergy-Related Immune Responses by Helminths. Journal of Biomedicine and Biotechnology, 2011, Article ID: 821578. http://dx.doi.org/10.1155/2011/821578

[5] Flohr, C., Tuyen, L.N., Quinnell, R.J., Lewis, S., Minh, T.T., Campbell, J., et al. (2010) Reduced Helminth Burden Increases Allergen Skin Sensitization But Not Clinical Allergy: A Randomized, Double-Blind, Placebo-Controlled Trial in Vietnam. Clinical and Experimental Allergy, 40, 131-142.

[6] Cooper, P.J. (2002) Can Intestinal Helminth Infections (Geohelminths) Affect the Development and Expression of Asthma and allergic Disease? Clinical and Experimental Immunology, 128, 398-404. http://dx.doi.org/10.1046/j.1365-2249.2002.01908.x

[7] Holt, P.G., Macaubas, C., Stumbles, P.A. and Sly, P.D. (1999) The Role of Allergy in the Development of Asthma. Nature, 402, B12-B17. http://dx.doi.org/10.1038/35037009

[8] Barnes, P.J. (2008) The Cytokine Network in Asthma and Chronic Obstrcutive Pulmonary Disease. Journal of Clinical Investigation, 118, 3546-3556. http://dx.doi.org/10.1172/JCI36130

[9] Chung, K.F. and Barnes, P.J. (1999) Cytokines in Asthma. Thorax, 54, 825-857. http://dx.doi.org/10.1136/thx.54.9.825

[10] Araujo, M.I., Lopes, A.A., Medeiros, M., Cruz, A.A., Sousa-Atta, L. and Sole, D., et al. (2000) Inverse Association between Skin Response to Aeroallergens and Schistosoma mansoni Infection. International Archives of Allergy and Immunology, 123, 145-148. http://dx.doi.org/10.1159/000024433

[11] Cooper, P.J. (2004) The Potential Impact of Early Exposures to Geohelminth Infections on the Development of Atopy. Clinical Review in Allergy and Immunology, 26, 5-14. http://dx.doi.org/10.1385/CRIAI:26:1:5

[12] Wills-Karp, M., Santeliz, J. and Karp, C.L. (2001) The Germless Theory of Allergic Disease: Revisiting the Hygiene Hypothesis. Nature Review Immunology, 1, 69-75. http://dx.doi.org/10.1038/35095579

[13] Yazdanbakhsh, M., Kremsner, P.G. and van Ree, R. (2002) Allergy, Parasites, and the Hygiene Hypothesis. Science, 296, 490. http://dx.doi.org/10.1126/science.296.5567.490

[14] Hasan, M.M., Gofin, R. and Bar-Yishay, E. (2000) Urbanization and the Risk of Asthma among Schoolchildren in the Palestinian Authority. Journal of Asthma, 37, 353-360. http://dx.doi.org/10.3109/02770900009055459

[15] Maia, J.G., Marcopito, L.F., Amaral, A.N., Tavares Bde, F. and Santos, F.A. (2004) [Prevalence of Asthma and Asthma Symptoms among 13 and 14-Year-Old Schoolchildren, Brazil]. Revista de Saúde Pública, 38, 292-299. http://dx.doi.org/10.1590/S0034-89102004000200020

[16] van den Biggelaar, A.H., van Ree, R., Rodrigues, L.C., Lell, B., Deelder, A.M. and Kremsner, P.G., et al. (2000) Decreased Atopy in Children Infected with Schistosoma Haematobium: A Role for Parasite-Induced Interleukin-10. Lancet, 356, 1723-1727. http://dx.doi.org/10.1016/S0140-6736(00)03206-2

[17] Allen, J.E. and Wynn, T.A. (2011) Evolution of Th2 Immunity: A Rapid Repair Response to Tissue Destructive Pathogens. PLoS Pathogens, 6, Article ID: e1002003. http://dx.doi.org/10.1371/journal.ppat.1002003 
[18] Girgis, N.M., Gundra, M.U. and Loke, O. (2013) Immune Regulation during Helminth Infections. PLoS Pathogens, 9, Article ID: 1003250.

[19] Oluwole, O., Arinola, G.O., Falade, A.G., Ige, O.M., Falusi, A.G., Huo, D., et al. (2013) Allergy Sensitization and Asthma among 13- and 14-Year Old Schoolchildren in Southwestern Nigeria. African Health Sciences, 13, 144-153.

[20] Ngoc, P.L., Gold, D.R., Tzianabos, A.O., Weiss, S.T. and Celedon, J.C. (2005) Cytokines, Allergy, and Asthma. Current Opinion in Allergy and Clinical Immunology, 5, 161-166. http://dx.doi.org/10.1097/01.all.0000162309.97480.45

[21] Romagnani, S. (2004) Immunologic Influences on Allergy and the TH1/TH2 Balance. Journal of Allergy and Clinical Immunology, 113, 395-400. http://dx.doi.org/10.1016/j.jaci.2003.11.025

[22] Barnes, P.J. (2002) Th2 Cytokines and Asthma: An Introduction. Respiratory Research, 2, 64-65. http://dx.doi.org/10.1186/rr39

[23] Bach, J.F. (2002) The Effect of Infections on Susceptibility to Autoimmune and Allergic Diseases. New England Journal of Medicine, 347, 911-920. http://dx.doi.org/10.1056/NEJMra020100

[24] Dillon, S.R., Sprecher, C., Hammond, A., Bilsborough, J., Rosenfeld-Franklin, M., Presnell, S.R., et al. (2004) Interleukin 31, a Cytokine Produced by Activated T Cells, Induces Dermatitis in Mice. Nature Immunology, 5, 752-760. http://dx.doi.org/10.1038/ni1084

[25] Finkelman, F.D., Shea-Donohue, T., Morris, S.C., Gildea, L., Strait, R., Madden, K.B., et al. (2004) Interleukin-4- and Interleukin-13-Mediated Host Protection against Intestinal Nematode Parasites. Immunology Review, 201, 139-155. http://dx.doi.org/10.1111/j.0105-2896.2004.00192.x

[26] Fort, M.M., Cheung, J., Yen, D., Li, J., Zurawski, S.M., Lo, S., et al. (2001) IL-25 induces IL-4, IL-5, and IL-13 and Th2-Associated Pathologies in Vivo. Immunity, 15, 985-995. http://dx.doi.org/10.1016/S1074-7613(01)00243-6

[27] Harnett, W. and Harnett, M.M. (2006) Molecular Basis of Worm-Induced Immunomodulation. Parasite Immunology, 28, 535-543. http://dx.doi.org/10.1111/j.1365-3024.2006.00893.x

[28] Wang, L.J., Cao, Y. and Shi, H.N. (2008) Helminth Infections and Intestinal Inflammation. World Journal of Gastroenterology, 14, 5125-5132. http://dx.doi.org/10.3748/wjg.14.5125

[29] Barbu, A.E. and Pecht, I. (2005) Desensitization of Mast Cells' Secretory Response to an Immuno-Receptor Stimulus. Immunology Letter, 100, 78-87. http://dx.doi.org/10.1016/j.imlet.2005.06.009

[30] Sancho-Serra Mdel, C., Simarro, M. and Castells, M. (2011) Rapid IgE Desensitization Is Antigen Specific and Impairs Early and Late Mast Cell Responses Targeting FcepsilonRI Internalization. European Journal of Immunology, 41, 1004-1013. http://dx.doi.org/10.1016/j.imlet.2005.06.009

[31] Akdis, M., Verhagen, J., Taylor, A., Karamloo, F., Karagiannidis, C., Crameri, R., et al. (2004) Immune Responses in Healthy and Allergic Individuals Are Characterized by a Fine Balance between Allergen-Specific T Regulatory 1 and T Helper 2 Cells. Journal of Experimental Medicine, 199, 1567-1575. http://dx.doi.org/10.1084/jem.20032058

[32] Smits, H.H., Everts, B., Hartgers, F.C. and Yazdanbakhsh, M. (2010) Chronic Helminth Infections Protect against Allergic Diseases by Active Regulatory Processes. Current Allergy and Asthma Report, 10, 3-12. http://dx.doi.org/10.1007/s11882-009-0085-3 